\title{
Criteria of Fractal Reconstruction and Suppressing Background Events with the SePaC Method
}

\author{
T. G. Dedovich ${ }^{a, b}$ and M. V. Tokarev ${ }^{a, b}, *$ \\ a Joint Institute for Nuclear Research, Dubna, Russia \\ ${ }^{b}$ State University Dubna, Dubna, Russia \\ *e-mail: tokarev@jinr.ru
}

Received July 12, 2020; revised August 4, 2020; accepted August 5, 2020

\begin{abstract}
This work analyzes the $\mathrm{SePaC}$ method of fractals and various background events (mixed, random, and distributed according to Gauss's law, as well as by power and exponential laws) in order to search and reconstruct fractal structures. It is shown that some of the background events are reconstucted as fractals. Dimensional distributions of various events are considered. It is found that these distributions for fractals and background events are different. Characteristics are proposed that describe the features of fractal structures. They are compared for fractals and background events. Criteria for background suppression are proposed and the results of their application are presented. It is found that the application of the criteria changes the distribution of background events by dimension, and the use of a combination of criteria suppresses the background.
\end{abstract}

Keywords: fractal analysis, fractal dimension

DOI: $10.1134 / \mathrm{S} 1547477121010040$

\section{INTRODUCTION}

In the interactions of hadrons and high-energy nuclei, various processes take place that lead to the multiple production of particles. These include the hard scattering of quarks and gluons, parton showers in the initial and final states, multiparticle interactions, hadronization, and the decay of unstable hadrons. The theoretical description of these processes underlies the Monte Carlo generators of particle interactions. So, in the PYTHIA program [1], the parton cascade is a self-similar process with an ordered angular distribution, and the fragmentation of a string is described by branches developing according to the same scenario: the string passes into a hadron and a residual string, etc. In each subprocess, energy and momentum are distributed among the fission products according to probabilistic laws. It is assumed that such self-similar processes can lead to a fractal distribution of particles in the phase space. Distinctive properties of fractals are the inequality of fractal and topological dimensions (see Appendix A), scale invariance, and the power-law dependence of the number of structural elements on their size. For a natural fractal, self-similarity and power-law dependences are performed within the characteristic scales.

Note that the spectra of particle production in interactions of hadrons and nuclei are often described by a combination of power and exponential dependences [2]. In the region of small transverse momenta, the exponential law is fulfilled and, in the region of large transverse momenta, the power law is fulfilled. The first reflects the thermodynamic properties of the system and the second reflects the exchange character of the interaction of individual constituents. Recently, the C. Tsallis distribution [3-6] has been used to approximate the spectra. This parameterization makes it possible to simultaneously describe both power-law and exponential regimes. The shape of the distribution is determined by three parameters: "chemical potential," "temperature," and the parameter $q$ related to the relative temperature dispersion. Introduction $q$ is of fundamental importance in describing systems where Boltzmann statistics are not applicable. It is often referred to as the entropy index. It controls the nonextensiveness (nonadditivity) of Tsallis entropy. Temperature is the average of all Boltzmann temperatures and is also related to the Tsallis entropy and the internal energy of the system. With values $q \approx 1$, Tsallis parameterization corresponds to the Maxwell-Boltzmann distribution. In this case, temperature and chemical potential coincide with the corresponding quantities in Boltzmann thermodynamics. In the region of large transverse momenta, the experimental spectra exhibit a power-law behavior, which is well described by the Tsallis distribution at a chemical potential equal to zero. The main motivation for the introduction of Tsallis entropy [7, 8] was the applicability of generalized statistics obtained on its basis to 
fractals and multifractal systems. Note that various characteristics of complex phenomena demonstrate power-law behavior reflecting the hierarchical or fractal structure of objects and processes [9-14].

In collisions of high-energy protons and nuclei, there are events with a large multiplicity, and the spectra of particles can be described by various functions. This allows one to carry out an event-by-event analysis and investigate the question: do events with such spectra have fractal structures? If fractal events are detected, their distribution in dimension $D_{F}$ can be investigated. It is assumed that the change of $D_{F}$ may be associated with phase transitions. In [15] it is indicated that the manifestation of fractality (intermittency) is possible due to the phase transition of quarks to hadrons. This point of view is reflected in studies of a two-dimensional lattice of spins (Ising model), in which the intermittency (fractal) behavior of the system occurs at the phase transition temperature and the intermittency exponents (dimension) are related to the critical exponents of the system under study [16].

In a fractal analysis of events, the method of determining dimension $D_{F}$ is of great importance. In papers [17, 18], $\mathrm{PaC}$ and $\mathrm{SePaC}$ methods of analysis are presented. They were used to reconstruct the characteristics of various types of fractals [19-22]. In addition to dimension, these methods allow you to determine the number of levels $N_{\text {lev }}$, the basis of formation $P_{F}$, and the structure of fractals. The number of levels is related to the number of different scales at which scale invariance manifests itself. The two-stage analysis procedure developed in [23] made it possible to increase the efficiency of recovery $D_{F}, N_{\text {lev }}$ and $P_{F}$ in data sets containing different kinds of fractals. The advantage of the two-step procedure of the $\mathrm{SePaC}$ method over $\mathrm{PaC}$ and the widely used box-counting method $(B C$ method) is shown [24, 25].

In this paper, we propose and study the criteria for separating fractal and background events (mixed, random, and distributed according to Gauss's law, as well as according to power and exponential laws). Section 1 describes the data sets used in the work. Sections 2 and 3 provide a brief description of the $\mathrm{SePaC}$ method and justified the choice of parameters. Section 4 shows the distributions of events reconstructed as fractals in dimension $D_{F}$. In Section 5, the characteristics describing the formation of fractals are investigated and, on their basis, criteria for the suppression of various background events are introduced. Section 6 shows the results of applying the introduced criteria for various data sets. The main conclusions are formulated in the Conclusions.

\section{DESCRIPTION OF DATA SETS}

A numerical experiment is carried out in this work. The generated data sets (fractals and various background events) are analyzed using the $\mathrm{SePaC}$ method for the search and reconstruction of fractal structures. All sets contain 1823 events. Every Event $=\left\{x_{1}, x_{2}, \ldots, x_{n}\right\}$ event includes $n$ points with coordinates $x_{i}$, where $x$ is the investigated quantity.

The set of fractals contains fractals with independent [19], dependent [20], and combined division [21]. They differ in the number of points (from 8 to 1024), the basis of formation $P_{F}$ (from 3 to 8 ), and structures $S$ (see Appendix B).

Mixed, random, and dynamic events are used as background events.

Mixed events do not contain duplicate structures. They are derived from fractals with the following requirements:

(i) the distribution by the number of points coincides with the original

(ii) all points of the original set are used (and only them)

(iii) each event contains points from different initiating events

(iv) each event is formed from initial events having the same or less multiplicity.

In dynamic events, the distribution over the studied value $x$ are described by exponential $\operatorname{Exp}(k x)$ and power law $\operatorname{Pow}(x, k)$ dependences, as well as the Gaussian distribution $\operatorname{Gaus}(\sigma)$, where $\sigma$ is the standard deviation. For fractals, dynamic, and random events, the distribution by the number of points is the same.

\section{SePaC FRACTAL ANALYSIS METHOD}

This section provides a brief description of the method of systems of equations of power-law coverings $(\mathrm{SePaC})$.

The $\mathrm{SePaC}$ method is based on the definition of the Hausdorff-Besicovitch dimension (see Appendix A), according to which different sets of coverings are considered to calculate the dimension. The distribution of the event over the studied value $x$ is used as a coverage. Distribution boundaries can be selected in different ways (minimum/maximum in an event, minimum/maximum in a data block, or fixed values). The number of bins $\left\{M_{i}\right\}$ in the distributions of each set changes according to the power law $M_{i}=\left(P_{\text {cov }}\right)^{i}$, where $i=0,1,2 \ldots, l e v$. Value lev is determined from the condition of saturation of the number of nonzero bins in the distributions. Coverage sets differ in the value of the basis, which takes values $P_{\text {cov }}=3, \ldots, P_{\text {Max }}$ where $P_{\mathrm{Max}}$ is method parameter. For each coverage, different fractal formation hypotheses are tested. For this, a system of equations is constructed:

$$
\sum_{j=1}^{K_{i}}\left(l_{j i}\right)^{D_{i}}=1
$$


where $K_{i}$ is the number of fractal elements per $i$-th level and $l_{j i}$ is the length of the $j$ element on the $i$-th level. Dimension $D_{i}$ is calculated for each equation. A fractal element is considered either a nonzero bin (hypothesis of independent division) or a segment consisting of nonzero bins (hypothesis of dependent division). According to the location of nonzero bins, the structure $S_{k}$ of each $k$ - th element of the fractal is defined. For each level, the following condition is checked:

$$
\frac{\left|D_{i}-\langle D\rangle\right|}{\langle D\rangle}<\operatorname{Dev}
$$

where Dev is the method parameter and $\langle D\rangle$ is the average value of dimensions $D_{i}$. All levels for which this condition is fulfilled are called confidence levels and are used to determine the fractal dimension. Levels that do not satisfy condition (2) contain a large number of nondividing elements. A set of points is considered a fractal if there are at least three confidence levels. In this case, the basis of formation of $P_{F}=P_{\text {cov }}$, the number of overall $N_{\text {lev }}=$ lev and confidential $N_{\text {con }}$ levels, and structure $S_{k}$ and dimension $D_{F}=\langle D\rangle$ fractal is determined.

A more detailed description and features and results of using the $\mathrm{SePaC}$ method for different types of fractals are presented in [18-23]. Note that the twostep analysis procedure [23] allows one to reconstruct fractals accurately and approximately. At the first step, the following constraint is used:

$$
N_{i}=\left(N_{1}\right)^{i},
$$

where $N_{1}$ and $N_{i}$ are the number of fractal elements on the first and $i$-th level, respectively. In this case, fractals with independent and dependent divisions are reconstructed accurately. At the second step (without constraint), the restoration occurs approximately.

\section{PARAMETER SELECTION $\mathrm{SePaC}$ METHOD}

The $\mathrm{SePaC}$ method has two parameters: the base of the maximum power coverage $P_{\mathrm{Max}}$ and the maximum relative deviation $D e v$ fractal dimensions of various levels from their mean. With decreasing value Dev, the accuracy of fractal reconstruction is increased. Value $P_{\mathrm{Max}}$ depends on the data block. If the data contains fractals with the basis of formation $P_{F} \leq k$, then the value $P_{\text {Max }} \geq k$. With increasing parameter $P_{\mathrm{Max}}$, the portion of random events recovered as fractals increases [26]. Therefore, if the data sets contains background events and fractals with a large formation basis value, then the choice of value $P_{\text {Max }}$ to separate different types of data is not a trivial task.

\subsection{Analysis of Fractals}

In this paper, an approximate reconstruction of fractals is considered, so the data set is analyzed by the $\mathrm{SePaC}$ method without restriction (3). Distribution boundaries are selected at the minimum/maximum in the event.

Figure 1a shows the dependence of the portion Prtn of reconstructed fractals on parameter Dev. A wide range of coveriges is considered (the value of the base $P_{c o v}$ ranges from 3 to $P_{\text {Max }}=100$ ), which makes it possible to exclude the influence of parameter $P_{\mathrm{Max}}$ on the dependence under study. The figure shows that, with an increase in value Dev, portion Prtn increases and reaches a plateau of Prtn $=0.96$ at Dev $=0.05$. The value of $D e v=0.05$ is selected for further analysis.

Figure $1 \mathrm{~b}$ shows the dependence of the portion Prtn of restored fractals (black line) on parameter $P_{\text {Max }}$ at $D e v=0.05$. This figure shows that, with an increase in the value $P_{\text {Max }}$, the portion rises and reaches a plateau at $P r t n=0.96$. Minimum value $P_{\text {Max }}$ on a plateau of 15 allows you to maximally restore fractals.

\subsection{Analysis of Various Background Events}

Next, consider the dependence (Figs. 1b, 1c, 1d) of the portion of Prtn events restored as fractals from parameter $P_{\text {Max }}$ at $D e v=0.05$ for various data set. Each type of data is depicted by a line of a certain color, which is indicated in brackets when describing figures.

Figure $1 \mathrm{~b}$ shows the dependence $\operatorname{Prtn}\left(P_{\mathrm{Max}}\right)$ for mixed (blue line) and random (red) data, as well as events distributed according to the Gaussian with $\sigma=0.3$ (green). Note that the behavior $\operatorname{Prtn}\left(P_{\mathrm{Max}}\right)$ for fractals and the presented background events differ significantly. The dependences for background events are similar. When $P_{\mathrm{Max}}=15$, the largest number of fractals is reconstructed $(96 \%)$ and fewer than $2 \%$ background events are restored as fractals. With an increase in this parameter, the portion of background events also increases as the value $P_{\text {Max }}=100$ reaches a maximum. For mixed data and Gaussian events, the maximum value Prtn equals 0.43 . It was found that the dependence $\operatorname{Prtn}\left(P_{\mathrm{Max}}\right)$ is weakly sensitive to choice $\sigma$ for Gaussian events. When $\sigma$ changes from 0.03 to 0.3 , the maximum values of Prtn differ by no more than $3 \%$. For random events, the maximum portion is 0.28 .

Thus, the choice of parameters Dev $=0.05$ and $P_{\text {Max }}=15$ allows us to restore fractals and suppress events distributed by the Gaussian, as well as mixed and random data.

This paper considers sets of events in which the investigated quantity $X$ is distributed according to the exponential and power law. For different values of parameters of these distributions, the portions of 

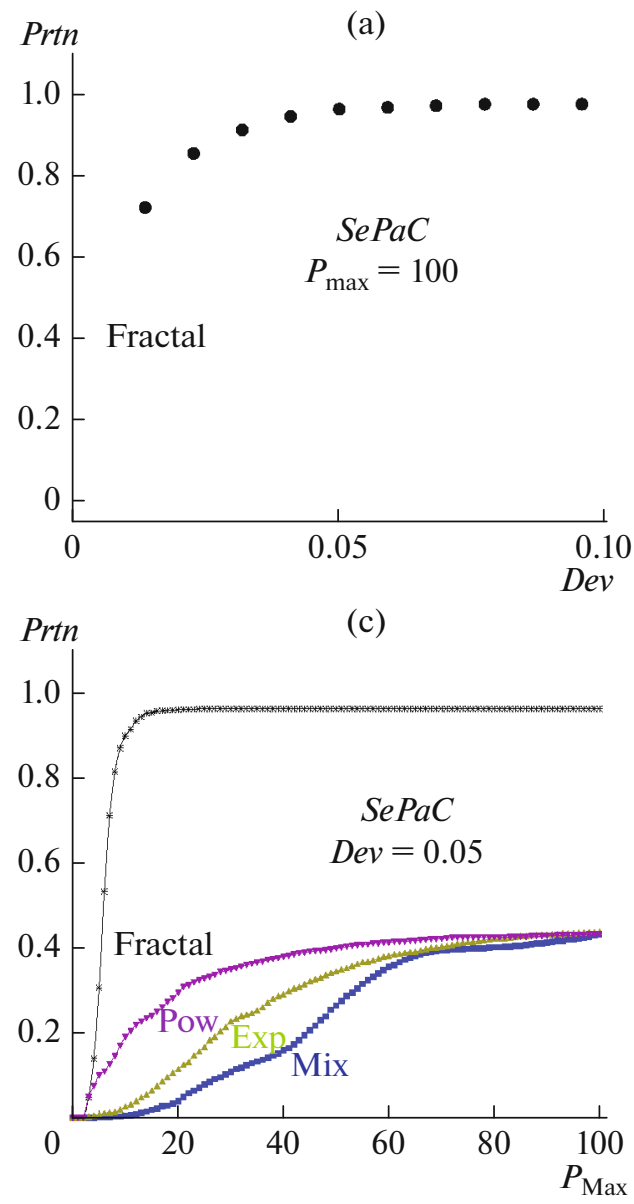

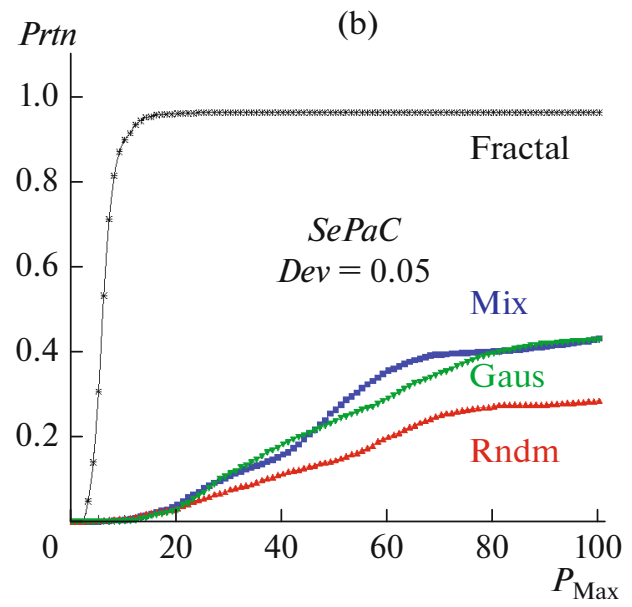

(d)

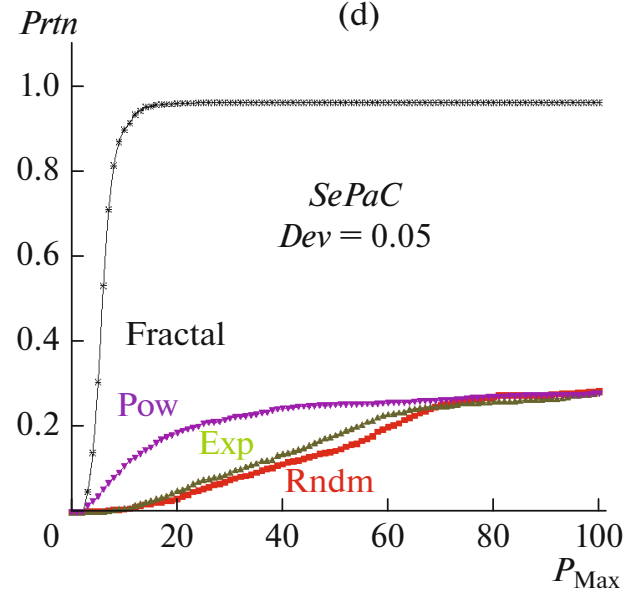

Fig. 1. Dependence of portion Prtn of events reconstructed as fractals from Dev at $P_{\text {Max }}=100$ for fractals (a) and from $P_{\text {Max }}$ at $D e v=0.05$ for different types of events $(\mathrm{b}, \mathrm{c}, \mathrm{d})$.

events reconstructed as fractals are different. Events described by distributions $\operatorname{Exp}(-1.2 x), \operatorname{Exp}(-0.35 x)$ and $\operatorname{Pow}(x,-2.04), \operatorname{Pow}(x,-0.984)$ were selected for analysis.

Figure 1c shows dependence $\operatorname{Prtn}\left(P_{\mathrm{Max}}\right)$ for $\operatorname{Exp}(-1.2 x)$ (green line) and Pow $(x,-2.04)$ (purple) distributions, as well as for mixed (blue) events. For all sets, the maximum value $\operatorname{Prtn}=0.43$. At $P_{\mathrm{Max}}=15$, $25 \%$ power and $7 \%$ exponential events are reconstructed.

Figure 1d shows dependence $\operatorname{Prtn}\left(P_{\mathrm{Max}}\right)$ for $\operatorname{Exp}(-0.35 x)$ (green line) and Pow $(x,-0.984)$ (purple) distributions and random (red) events. For all sets, the maximum value is $P r t n=0.28$. When $P_{\text {Max }}=15,15 \%$ power and $2 \%$ exponential events are reconstructed.

In Figs. $1 \mathrm{c}$ and $1 \mathrm{~d}$, it is seen that, at $P_{\mathrm{Max}} \leq 70$, the value of Prtn is largest for power distributions. When $P_{\text {Max }}>70$, the values of Prtn are approximately the same for all data.

It is shown that some of the background events are reconstructed as fractals. It was found that the sup- pression of power law and exponential events is less than that of mixed and random data, as well as events distributed over the Gaussian.

\section{DISTRIBUTION OF EVENTS BY FRACTAL DIMENSION $D_{F}$}

As was shown above, events reconstructed as fractals may contain impurities. Consider the distributions of various types of events by dimension $D_{F}$ shown in Fig. 2. Each type of data is depicted by an area of a certain color, which is indicated in brackets when describing figures.

Figure 2a shows the distributions over $D_{F}$ for fractals (gray area), mixed (blue) and random (red) data, and events distributed according to the Gaussian with $\sigma=0.3$ (green). The dimension of fractals varies from 0.3 to 0.9 . The rest of the distributions differ significantly from the fractal ones and have a pronounced maximum, the position of which depends on the type of data. The maximum number of random events has the dimension $D_{F}=0.91$. Note that the position of 

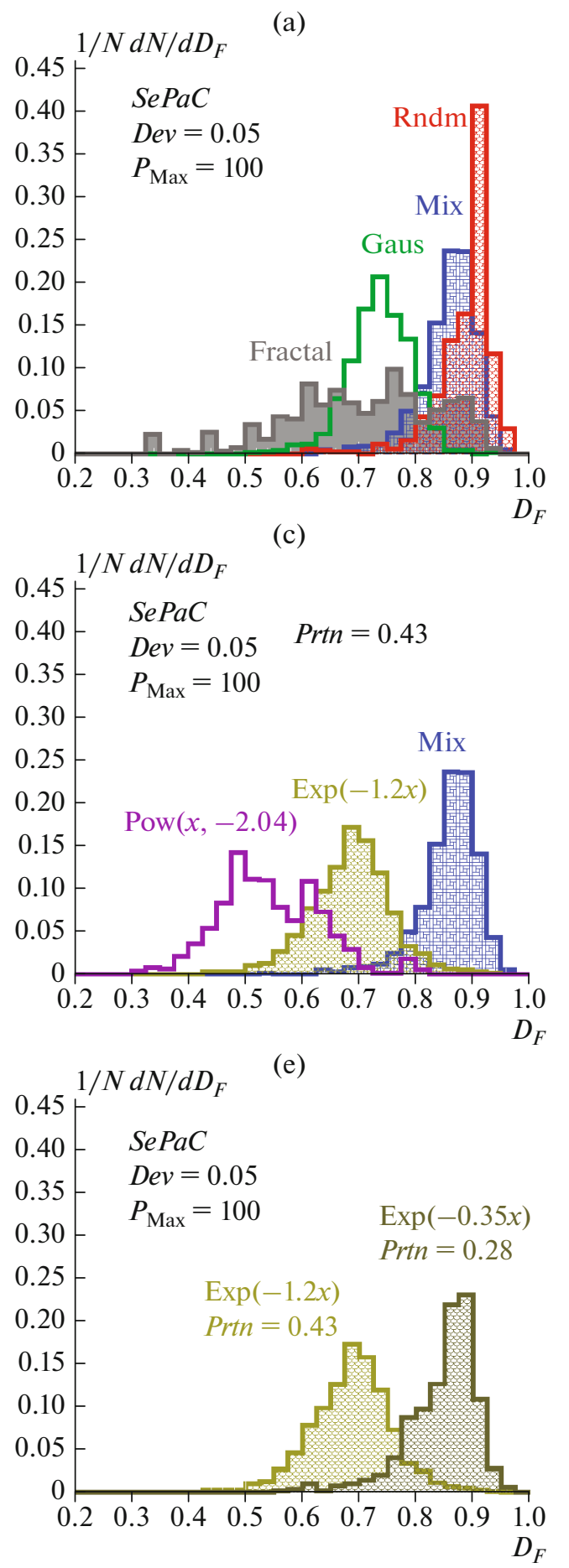

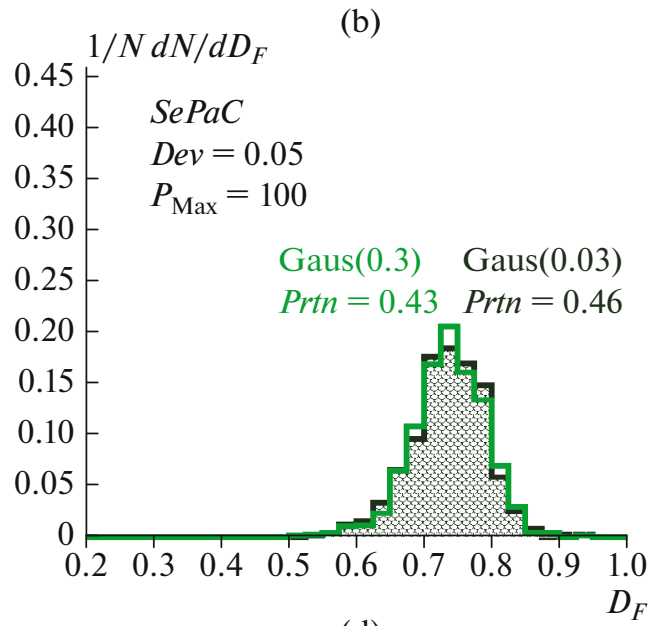

(d)

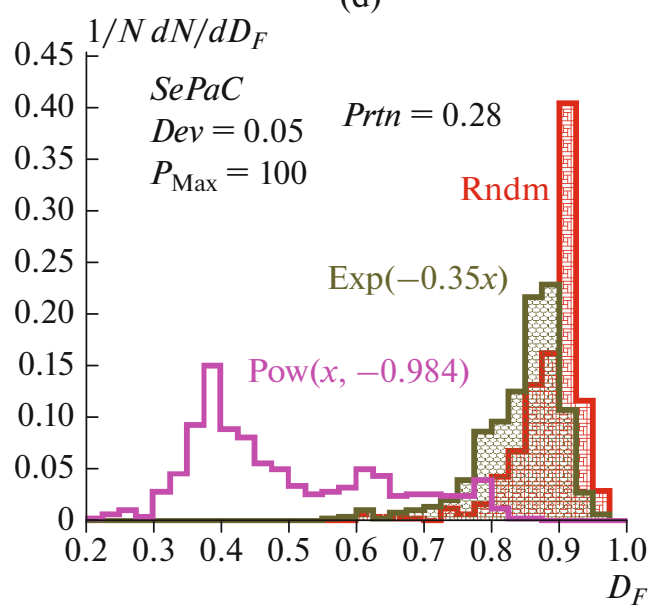

(f)

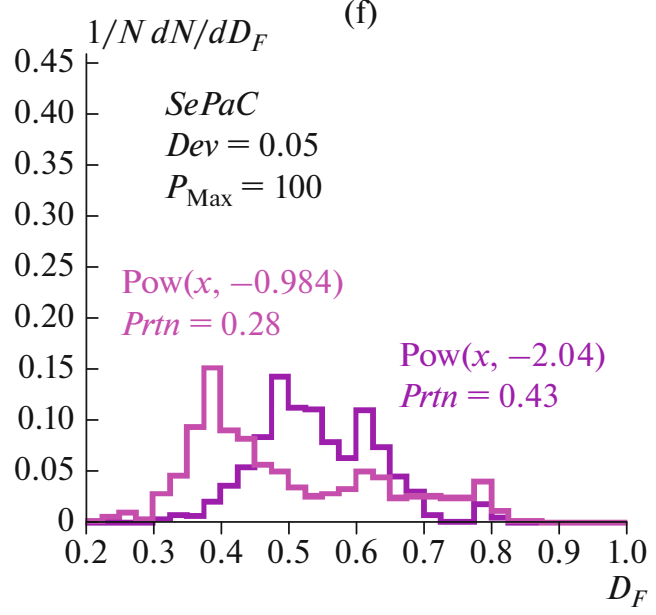

Fig. 2. Distribution of various events by fractal dimension $D_{F}$.

the maximum depends on the multiplicity distribution. Most mixed data and Gaussian events have a lower dimension value $\left(D_{F}=8.5-8.9\right.$ and $D_{F}=0.73$, respectively). The position of maxima for data sets $\operatorname{Gaus(0.3)}$ and Gaus(0.03) with different meanings $\sigma$ practically coincide (see Fig. 2b).
Figure 2c shows the distribution by $D_{F}$ for mixed (blue area) data, as well as exponential $\operatorname{Exp}(-1.2 x)$ (olive) and power law Pow $(x,-2.04)$ (purple) events, having the same maximum value Prtn $=0.43$. It can be seen that all distributions are different. Mixed data is shifted to high values of $D_{F}$. Power law events are 
characterized by three maxima at $D_{F}=0.49,0.61,0.79$ and the exponential one at $D_{F}=0.69$.

Figure $2 \mathrm{~d}$ shows distributions $D_{F}$ for random (reddish) data, as well as exponential $\operatorname{Exp}(-0.35 x)$ (olive) and power law $\operatorname{Pow}(x,-0.984)$ (purple) events having the same maximum value $\operatorname{Prth}=0.28$. The figure shows that all distributions are different, and random data is shifted to the region of large values $D_{F}$. Power law events are characterized by three maxima at $D_{F}=0.39,0.61,0.79$ and exponential events by one at $D_{F}=0.87-0.89$.

Exponential events $\operatorname{Exp}(-1.2 x), \operatorname{Exp}(-0.35 x)$ have different positions of maxima in the distribution over $D_{F}$ (see Fig. 2e). Power events Pow $(x,-2.04)$, Pow $(x,-0.984)$ differ in the position of the first maximum (see Fig. 2f), while the second and third coincide. Most power law events have a dimension value in the region of the first maximum.

Thus, the distributions in dimension $D_{F}$ for fractals and background events are different. The position of the maxima for mixed and random events depends on the multiplicity distribution; for exponential ones, it depends on the value of the exponential parameter; and, for events distributed according to the Gaussian, it does not depend on the value of $\sigma$. The distributions of power law events have three maxima; the position of the first depends on the exponent and the position of the others does not.

\section{CHARACTERISTICS OF THE FRACTAL FORMATION PROCESS}

In this section, we study the quantities that characterize the features of the process of formation of fractals. It is shown that the accuracy of reconstruction of fractal structures is determined by the values of the proposed quantities. These characteristics are compared for fractals and background events, and criteria for background suppression are proposed.

\subsection{Number of Fractal Elements at Different Levels}

When analyzing fractals by the basic $\mathrm{SePaC}$ method, the number of selected elements on the first and $i$-th level $\left(N_{1}\right.$ and $\left.N_{i}\right)$ are related by power dependence (3). The exponent is equal to the level number. In the case of an approximate reconstruction, the exponent takes on the value $K$.

$$
N_{i}=\left(N_{1}\right)^{K} \text {. }
$$

We determine the average relative deviation of the exponent $K$ in power law dependence (4) on the number of fractal level $i$ according to the formula

$$
\bar{\delta}_{K}=\frac{1}{N_{\text {con }}} \sum_{i=2}^{N_{\text {con }}} \frac{|K-i|}{i} .
$$

With the accurate reconstruction of fractals, the value $\bar{\delta}_{K}=0$. Figure 3 shows the distributions of various events in terms of $\bar{\delta}_{K}$.

In Fig. 3a, fractals are shown in gray, mixed and random data are shown in blue and red, and Gaussian events with $\sigma=0.3$ are green. For most fractals, $\bar{\delta}_{K}$ lies in the range $[-0.2,0.2]$ and, in the negative region, reaches the value $\bar{\delta}_{K}=-1.5$. The rest of the distributions differ significantly from the fractal ones. For most mixed and random events, the distribution over $\bar{\delta}_{K}$ lies in the region of positive values and reaches $\bar{\delta}_{K}=3.0$. The maximum number of Gaussian events corresponds to $\bar{\delta}_{K}=-0.6$. Peak positions for data sets $\operatorname{Gaus}(0.3)$ and $\operatorname{Gaus}(0.03)$ with different meanings $\sigma$ coincide (see Fig. 3b).

Figure $3 \mathrm{c}$ shows the distributions over $\bar{\delta}_{K}$ for mixed (blue area) data, as well as exponential $\operatorname{Exp}(-1.2 x)$ (olive) and power law $\operatorname{Pow}(x,-2.04)$ (purple) events having the same maximum value Prtn $=0.43$. Figure $3 \mathrm{~d}$ shows the distributions for $\bar{\delta}_{K}$ for random (red) data, as well as exponential $\operatorname{Exp}(-0.35 x)$ (olive) and power law Pow $(x,-0.984)$ (purple) events having the same maximum value Prtn $=0.28$. Distribution by $\bar{\delta}_{K}$ for power law events contains a peak in the region $[-0.2,0.2]$, and most mixed, random, and exponential data are outside the specified range.

Distributions for various exponential $\operatorname{Exp}(-1.2 x)$, $\operatorname{Exp}(-0.35 x)$ events are shifted to the area of negative and positive values $\bar{\delta}_{K}$, respectively (see Fig. 3e). Distributions for power law Pow $(x,-2.04)$, Pow $(x,-0.984)$ events are similar and lie in the area $[-1.0,1.0]$ (see Fig. 3f).

Therefore, the constraint $\left|\bar{\delta}_{K}\right| \leq 0.2$ can be used as a criterion for separating fractal and background events.

\subsection{The Number of New Elements in Each Act of Division}

Consider the selection of fractal elements in the case of their approximate reconstuction by the $\mathrm{SePaC}$ method.

Figure 4 shows the elements of the fractal at different levels. All elements are numbered consecutively, independent of the level. In Fig. 4a, the first element is divided into three parts $\left(N_{d 1}=3\right)$. At the next level, the second element forms more elements than the original $\left(N_{d 2}=4\right)$. The total number of such elements is denoted as $N_{+}$. The third and fourth elements form fewer elements than the original $\left(N_{d 3}=1, N_{d 4}=2\right)$. The total number of such elements is denoted as $N_{-}$.

We determine the average relative deviation $\bar{\delta}_{D}$ of number of divisions $N_{d j}$ from the original $N_{d 1}$ for ele- 
(a)

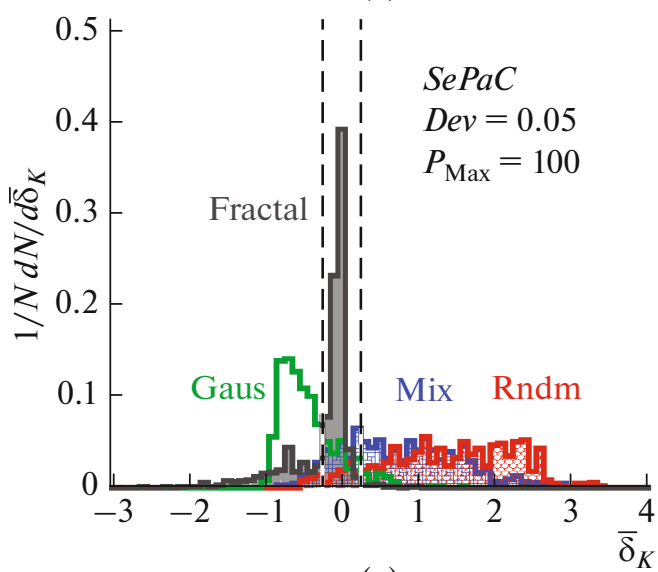

(c)

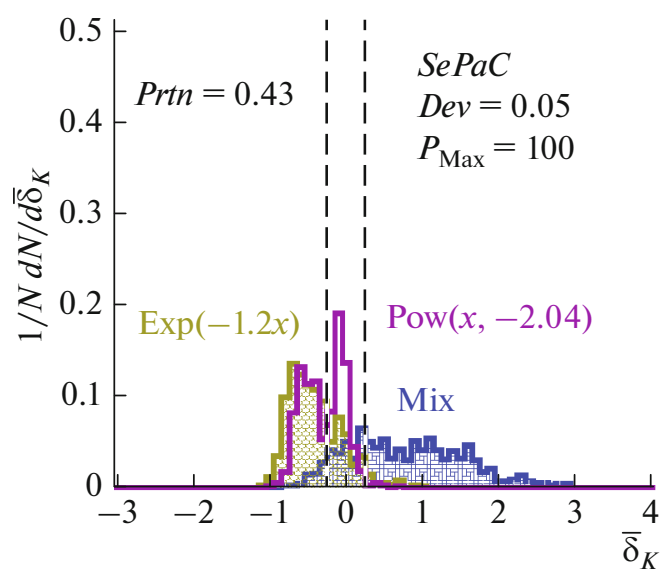

(e)

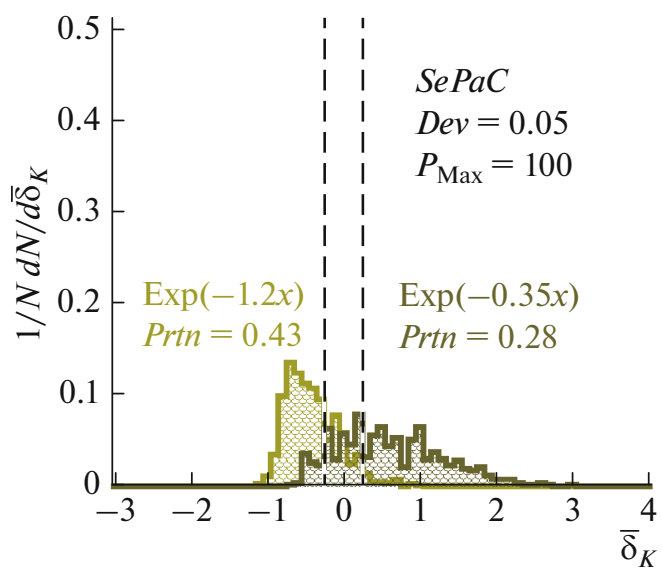

(b)

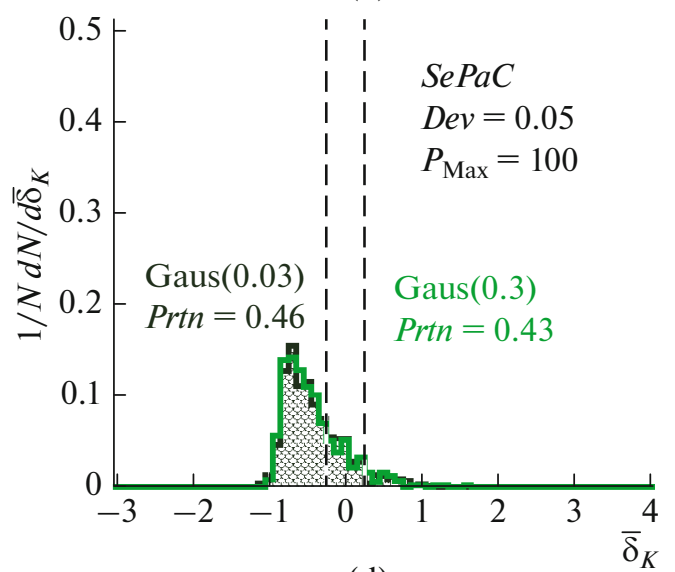

(d)

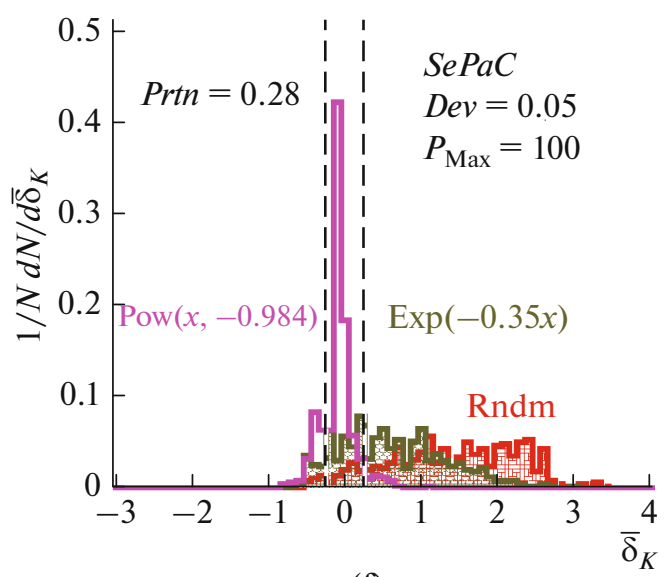

(f)

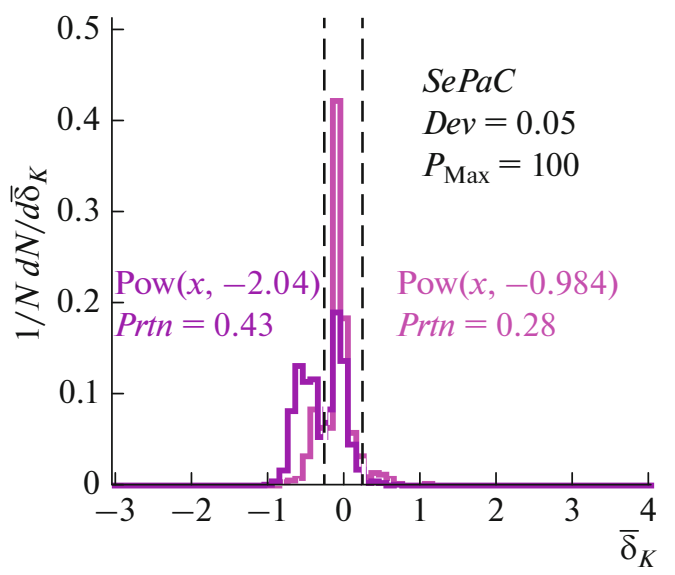

Fig. 3. Distribution of various events by $\bar{\delta}_{K}$.

ments with divisions greater $\bar{\delta}_{D+}$ and less $\bar{\delta}_{D-}$ in comparison with the original by the following formulas:

$$
\begin{aligned}
& \bar{\delta}_{D+}=\frac{1}{N_{+}} \sum_{i=2}^{N_{+}} \frac{N_{d j}-N_{d 1}}{N_{d 1}}, \\
& \bar{\delta}_{D-}=\frac{1}{N_{-}} \sum_{i=2}^{N_{-}} \frac{N_{d j}-N_{d 1}}{N_{d 1}} .
\end{aligned}
$$

With the accurate reconstruction of fractals, the values of $\bar{\delta}_{D+}$ and $\bar{\delta}_{D_{-}}$are equal to zero. Figure 5 shows the distributions for various events on $\bar{\delta}_{D}$.

In Fig. 5a fractals are shown in gray, mixed and random data are shown in blue and red, and Gaussian events with $\sigma=0.3$ are green. For most fractals, the distribution lies in the range $[-0.5,0.5]$. The rest of the distributions differ significantly from the fractal ones, 
(a)

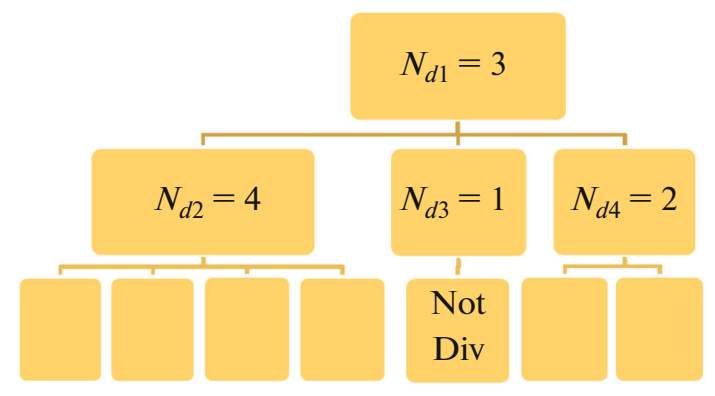

(b)

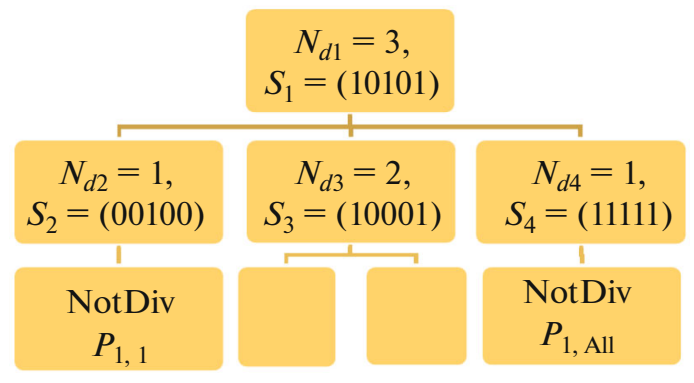

Fig. 4. Division scheme of the fractal elements at different levels in the case of approximate reconstruction.

especially in the area of positive values. Distributions for data sets $\operatorname{Gaus(0.3)}$ and $\operatorname{Gaus}(0.03)$ with different meanings $\sigma$ practically coincide (see Fig. $5 b$ ).

Figure $5 \mathrm{c}$ shows the distributions over $\bar{\delta}_{D}$ for mixed (blue area) data, as well as exponential $\operatorname{Exp}(-1.2 x)$ (olive) and power law $\operatorname{Pow}(x,-2.04)$ (purple) events having the same maximum value Prtn $=0.43$. Figure 5d shows the distributions $\bar{\delta}_{D}$ for random (red area) data, as well as exponential $\operatorname{Exp}(-0.35 x)$ (olive) and power law $\operatorname{Pow}(x,-0.984)$ (purple) events having the same maximum value Prtn $=0.28$. Most exponential and power law events lie outside the region $[-0.5,0.5]$.

Distributions for exponential events $\operatorname{Exp}(-1.2 x)$ and $\operatorname{Exp}(-0.35 x)$ (see Fig. 5e) have a slight difference among themselves. A similar feature is observed for power law Pow $(x,-2.04)$, Pow $(x,-0.984)$ (see Fig. 5f) events.

The constraint $\left|\bar{\delta}_{D}\right| \leq 0.5$ can be used as a criterion for separating fractal and background events.

\subsection{Portion of Non-Dividing Elements}

Let us consider the structure of fractal elements at different levels in the case of approximate reconstruction (see Fig. 5b). The first element is divided into three parts $\left(N_{d 1}=3\right)$ and has the structure $S=(10101)$. The second element is non-dividing $\left(N_{d 2}=1\right)$, because the structure $S=(00100)$ contains only one filled part. The portion of such elements at the confidence level is denoted by $P_{1,1}$. The third element is divided into two parts $\left(N_{d 3}=2\right)$, which is reflected in the structure $S=(10001)$. All parts of the fourth element are filled $S=(11111)$ and in the future it is not divided $\left(N_{d 4}=1\right)$. Let us denote the portion of such elements at the confidence level as $P_{1, A l l}$. With the accurate reconstruction of fractals, the values $P_{1,1}$ and $P_{1, \text { All }}$ are equal to zero. Figure 6 shows the distribution of events by portion $P_{1,1}$ for various data.
In Fig. 6a fractals are shown in gray, mixed and random data are shown in blue and red, and Gaussian events with $\sigma=0.3$ are green. For most fractals, the distribution lies in the range $[0.0,0.2]$. The rest of the distributions go beyond this region and have a peak at $P_{1,1}=0.6$. For data sets $\operatorname{Gaus}(0.3)$ and $\operatorname{Gaus}(0.03)$ with different values of $\sigma$, the distributions practically coincide (see Fig. 6b).

Figure 6c shows the distributions over $P_{1,1}$ for mixed (blue area) data, as well as exponential $\operatorname{Exp}(-1.2 x)$ (olive) and power law Pow $(x,-2.04)$ (purple) events having the same maximum value Prtn $=0.43$. Figure $6 \mathrm{~d}$ shows the distributions for $P_{1,1}$ for random (red) data, as well as exponential $\operatorname{Exp}(-0.35 x)$ (olive) and power law Pow $(x,-0.984)$ (purple) events having the same maximum value $\operatorname{Prtn}=0.28$. Most exponential and power law events lie outside the region [0.0,0.2].

Distributions for exponential events $\operatorname{Exp}(-1.2 x)$ and $\operatorname{Exp}(-0.35 x)$ (see Fig. 6e) have a slight difference between themselves. A similar feature is observed for power law Pow $(x,-2.04)$, Pow $(x,-0.984)$ (see Fig. 6f) events.

The constraint $P_{1,1} \leq 0.2$ can be used as a criterion for separating fractal and background events.

\section{DATA ANALYSIS BASED ON CRITERIA}

This section examines the influence of criteria on the number of events reconstructed by $\mathrm{SePaC}$ method as fractals, as well as on dimension distributions $D_{F}$ for various data. The following constraints are selected as criteria $K, D, P$ :

(i) $K$ : events with magnitude $\left|\bar{\delta}_{K}\right| \leq 0.2$

(ii) $D$ : events with magnitude $\left|\bar{\delta}_{D}\right| \leq 0.5$

(iii) $P$ : events with portion $P_{1,1} \leq 0.2$

The work considers all possible combinations of $K D, K P, D P$, and $K D P$ of these criteria. 
(a)



(c)

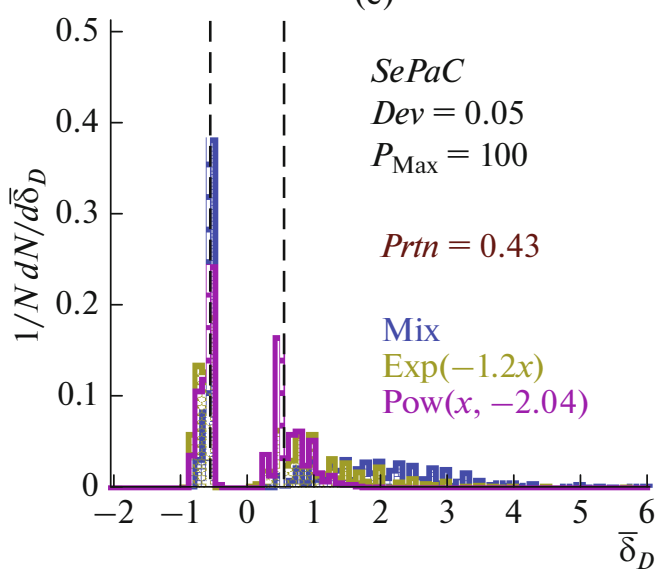

(e)

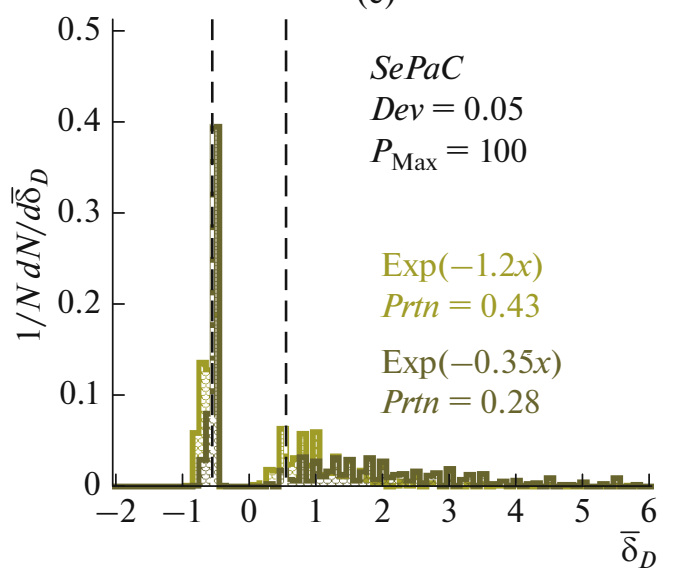

(b)

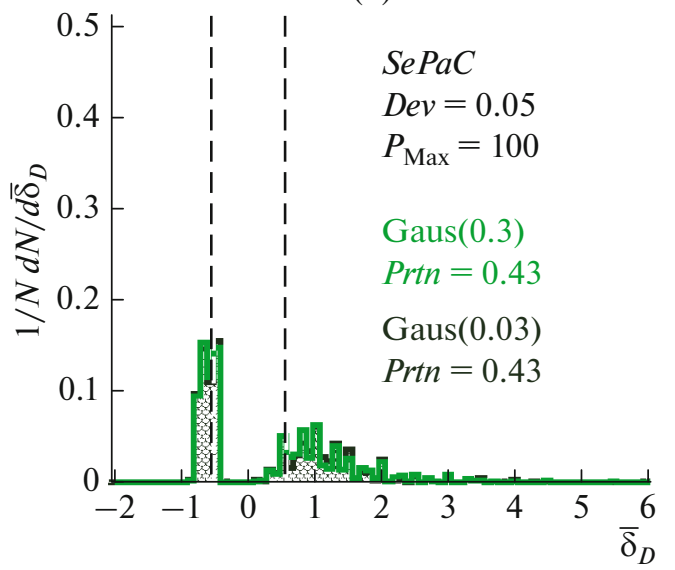

(d)

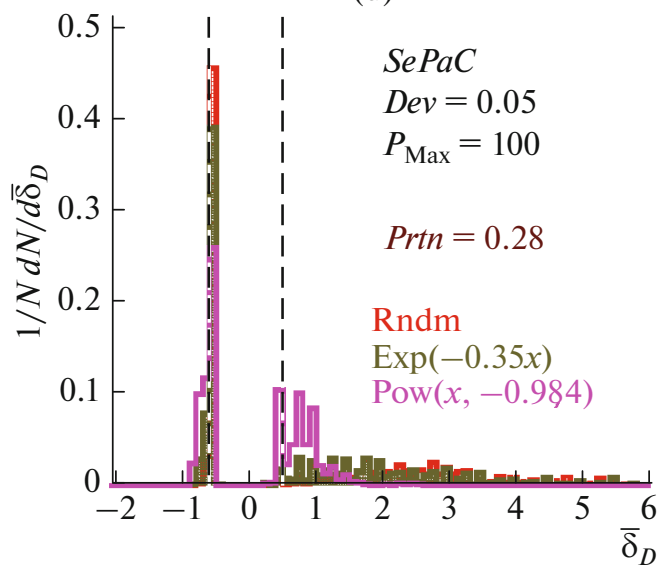

(f)

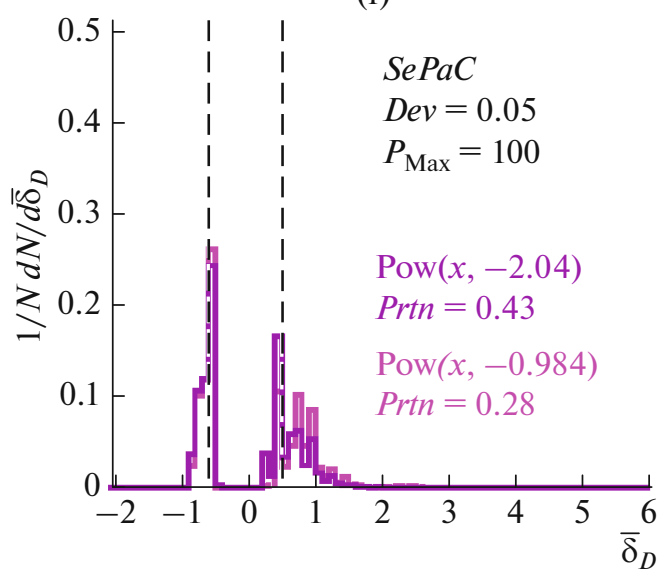

Fig. 5. Distribution of various events by $\bar{\delta}_{D}$.

\subsection{Reconstruction of Fractals and Suppression of Background Events}

In this section, fractals and various background events (mixed, random, Gaussian, exponential, and power law) are analyzed by the $\mathrm{SePaC}$ method using the proposed criteria.

Table 1 shows the portion of events (in percent) reconstructed as fractals after applying the criteria.
The value was determined in relation to the initial number of events (1823) in the data blocks. The second column shows the portion of events reconstructed without considering the criteria.

The Table 1 shows that the percentage of events reconstructed as fractals depends on the type of criterion applied. In the case of using single criteria, the greatest suppression occurs according to $K$ (71\% of fractals remain). The criteria are independent, since 

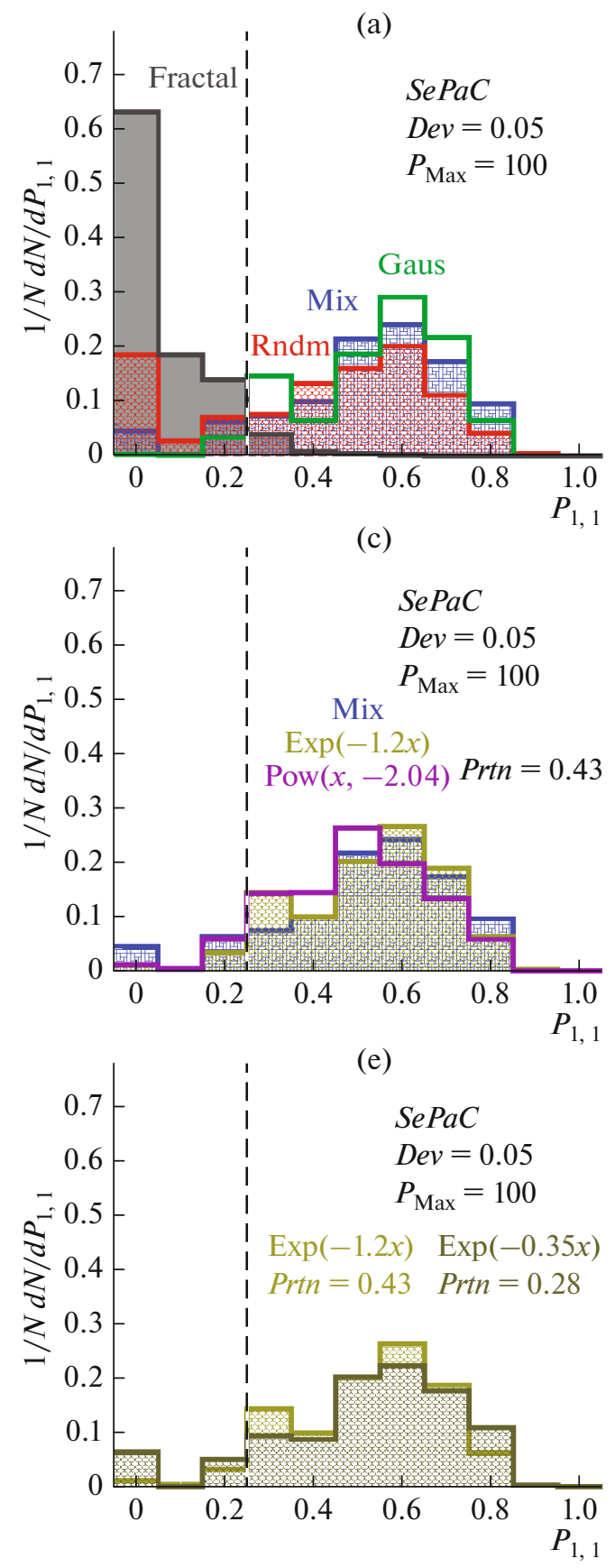
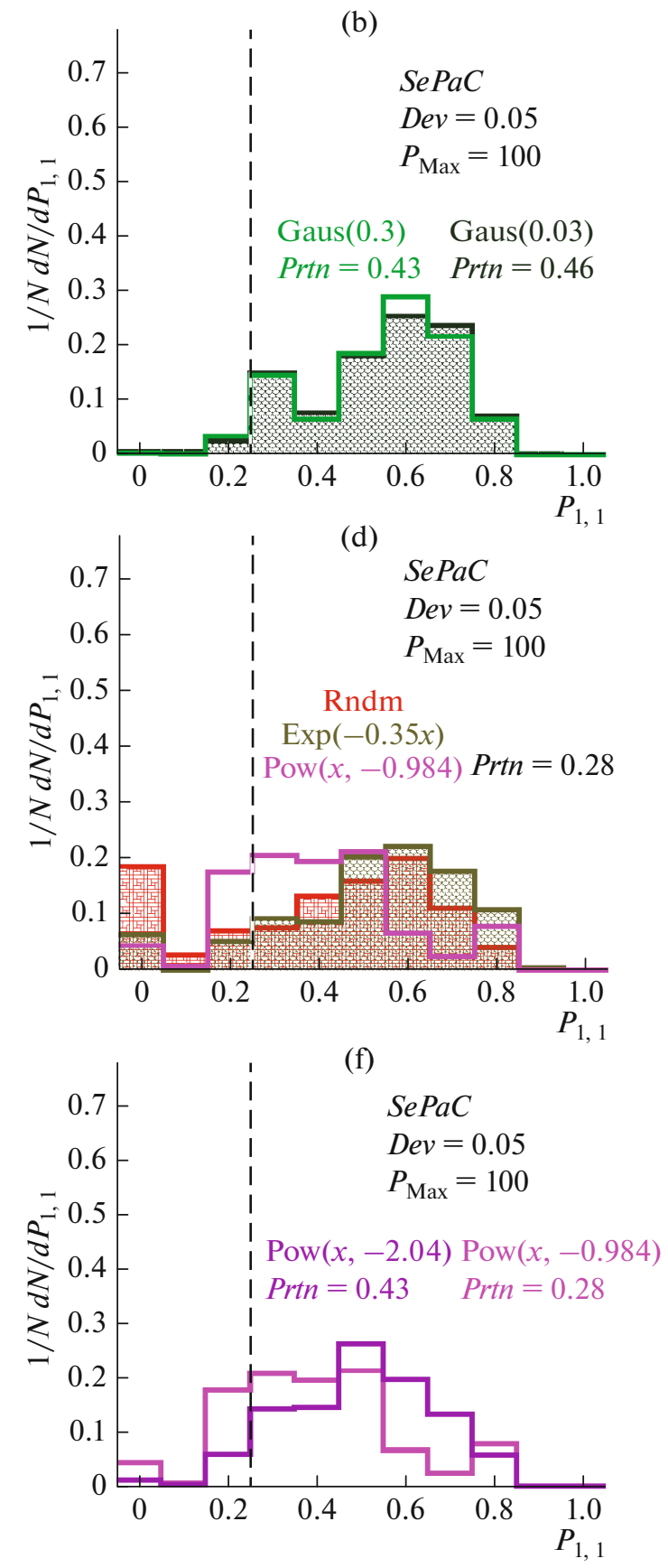

Fig. 6. Distribution of various events by portion $P_{1,1}$.

after applying any two of them together there are fewer events left than after applying them separately. Combination application $K D P$ suppresses half of the fractals (53.9\% remains).

Criterion $K$ leads to the greatest suppression of random data ( $1.8 \%$ remains) and has the lowest effect on power law events (more than $18.4 \%$ remains). Applying it to events distributed over Gaussian, as well as to mixed and exponential data, leaves from 5.4 to $8.4 \%$ of events.
Criterion $D$ leads to the greatest suppression of random, mixed, and exponential events $\operatorname{Exp}(-0.35, x)$ (remains less than 1.3\%) and has the least impact on power data $\operatorname{Pow}(x,-2.04)$ (12.6\% remains). Applying it to the rest of the sets leaves from 3.3 to $4.4 \%$ of events.

Criterion $P$ leads to the greatest suppression of events distributed over the Gaussian (remains less than $0.7 \%$ ) and has the least impact on random data (remains 6.7\%). Applying it to the rest of the sets 
Table 1. Portion of events reconstructed as fractals after the application of criteria

\begin{tabular}{l|c|r|r|r|r|r|r|c}
\hline \multirow{2}{*}{ Type events } & \% of events & \multicolumn{7}{c}{ \% of events after criteria applied } \\
\cline { 3 - 9 } & SePaC & \multicolumn{1}{c}{$K$} & \multicolumn{1}{c}{$D$} & $P$ & $K D$ & $K P$ & $D P$ & $K D P$ \\
\hline Fractals & 96 & 71.0 & 82.1 & 83.7 & 62.5 & 59.4 & 72.9 & 53.9 \\
Mixed & 43 & 7.3 & 1.2 & 3.1 & 0.5 & 0.4 & 0.3 & $<0.05$ \\
Random & 28 & 1.8 & 1.0 & 6.7 & 0.5 & 0.9 & 0.8 & 0.2 \\
Exp(-1.2) & 43 & 8.4 & 4.4 & 0.9 & 1.5 & 0.5 & 0.3 & 0.05 \\
Exp(-0.35) & 28 & 5.4 & 1.3 & 2.2 & 0.4 & 0.4 & 0.6 & 0.05 \\
Pow(x,-2.04) & 43 & 18.4 & 12.6 & 0.9 & 8.1 & 0.3 & 0.4 & 0.05 \\
Pow(x,-0.984) & 28 & 20.2 & 5.9 & 1.8 & 4.0 & 0.8 & 0.9 & 0.3 \\
Gaus(0.3) & 43 & 5.9 & 3.5 & 0.4 & 0.9 & 0.1 & 0.2 & 0.05 \\
Gaus(0.03) & 46 & 7.1 & 3.3 & 0.7 & 0.9 & 0.2 & 0.2 & $<0.05$ \\
\hline
\end{tabular}

leaves from 0.9 to $3.1 \%$ of events. Note that criterion $P$ most strongly affects background events and the least impact on fractals $(83.7 \%$ remains).

The combined application of the criteria enhances the suppression of background events. After applying combinations $K P$ and $D P$, less than $1 \%$ background events remains and, after $K D P$, less than $0.2 \%$.

Thus, the criteria applied to fractal events reduce the number of reconstructed fractals, and the applica- tion of several criteria can significantly suppress background events.

\subsection{Distribution by Fractal Dimension $D_{F}$}

Consider the change in the distributions of various events over $D_{F}$ after applying the criteria. Figures 7 and 8 correspond to events without (gray) and, after applying the criteria $K, D$, and $P$, blue, red, and green, respectively.
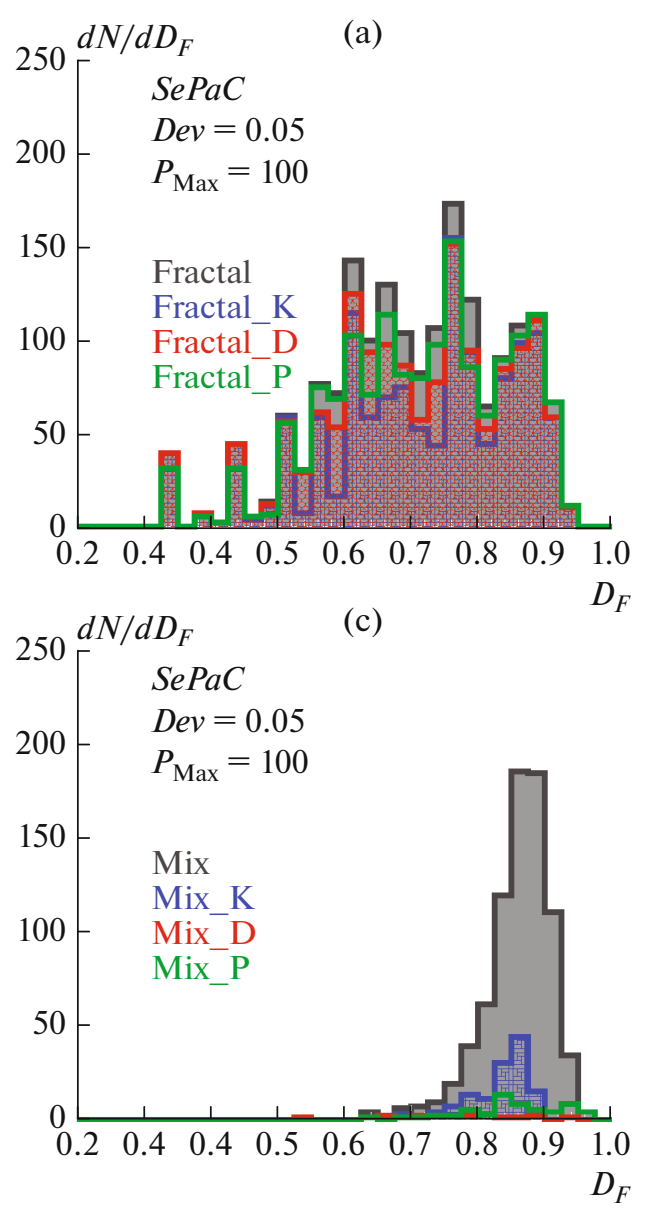
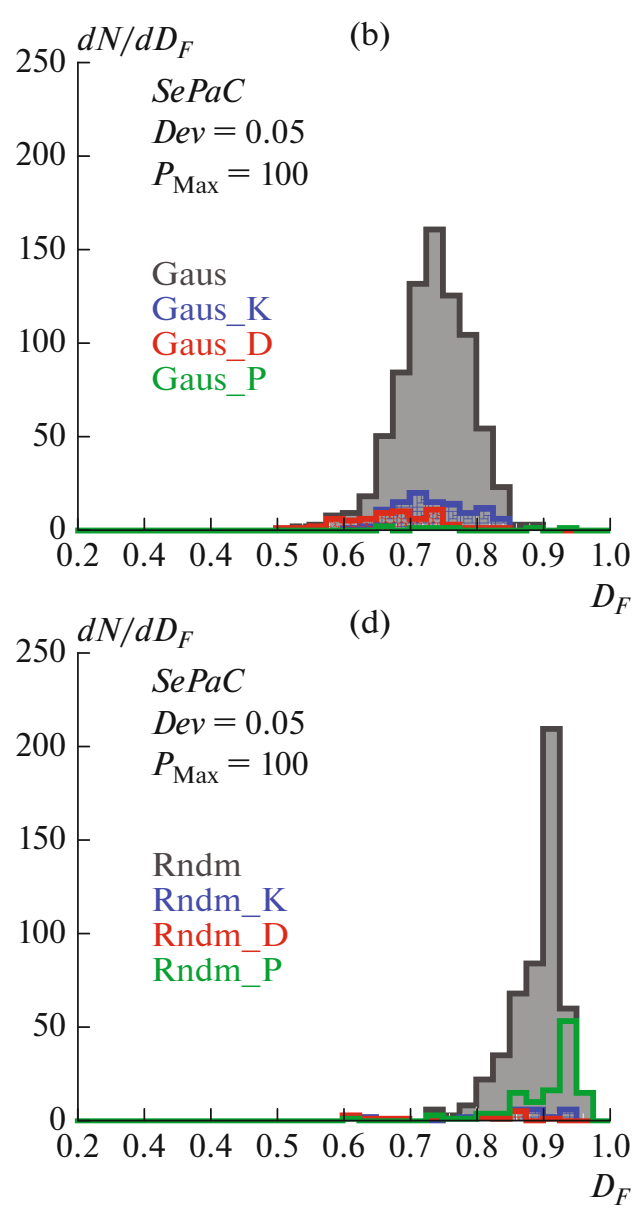

Fig. 7. Distribution by $D_{F}$ for fractals (a), Gaussian events (b), mixed (c), and random (d) data. 


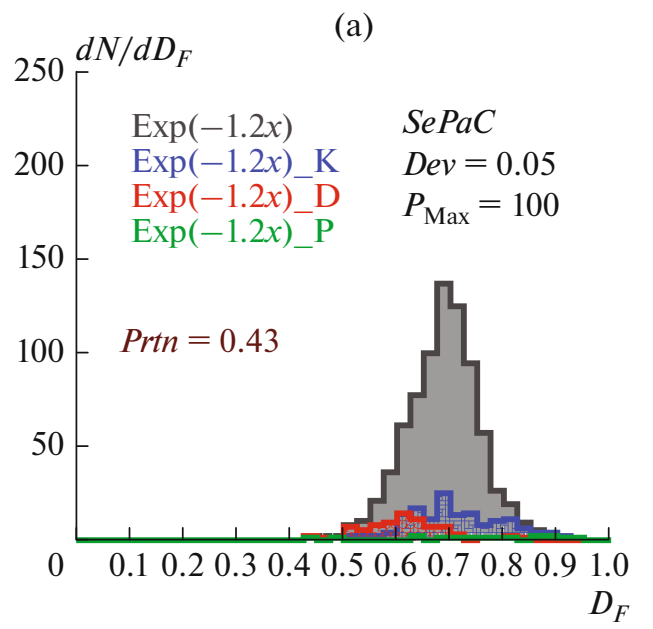

(c)

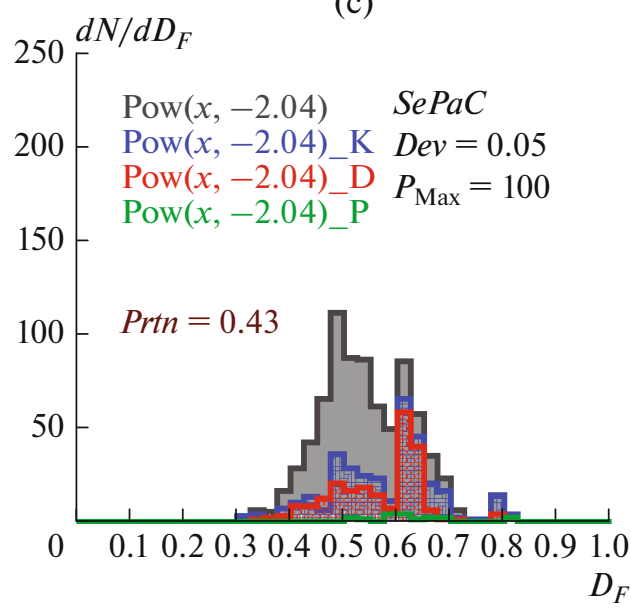

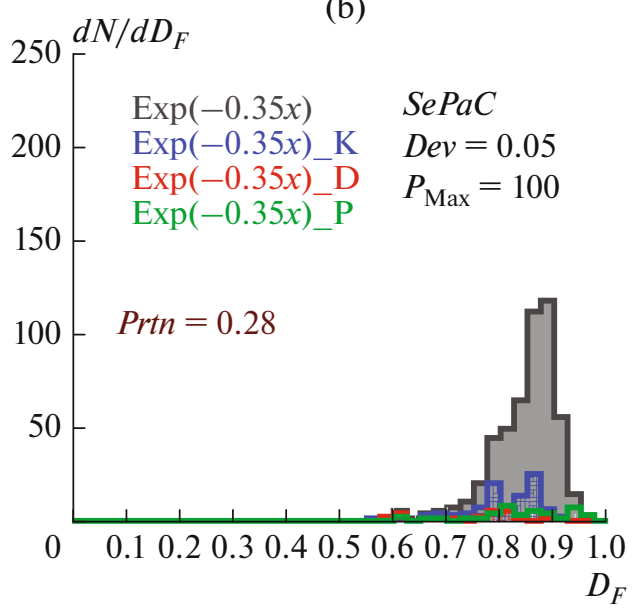

(d)

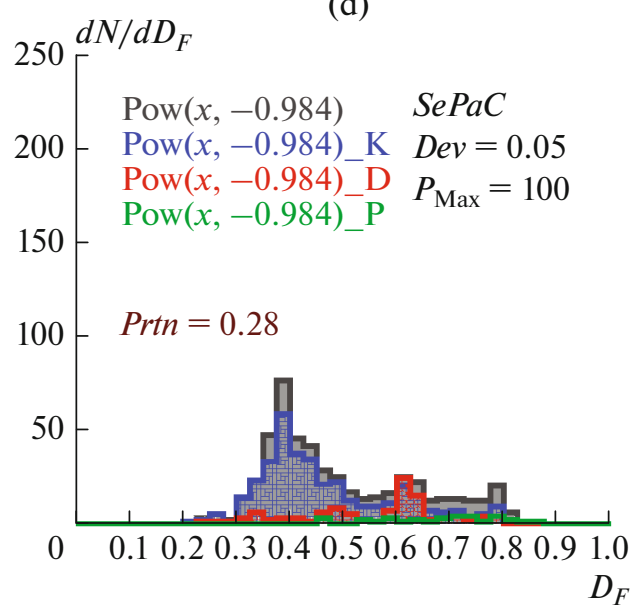

Fig. 8. Distribution by $D_{F}$ for exponential $(a, b)$ and power $(c, d)$ events.

Figure 7 shows the distribution over $D_{F}$ for fractals (a), Gaussian events with $\sigma=0.3$ (b), and mixed (c) and random (d) data. For fractals, taking into account the criteria insignificantly changes the shape of the distribution. The positions of the maxima remain at the same values $D_{F}$. For Gaussian events, applying criteria $K$ and $D$ significantly reduces the maximum, shifting it to the region of lower values $D_{F}$ and using $P$ suppresses distribution. For mixed events, taking into account criterion $K$ significantly reduces the maximum, and the use of $D$ and $P$ suppresses distribution. For random events, the application of criterion $P$ decreases the maximum, shifting it to the region of large values of $D_{F}$, and accounting for $K$ and $D$ suppresses distribution.

Figure 8 shows the distribution over $D_{F}$ for exponential $(\mathrm{a}, \mathrm{b})$ and power law (c, d) events. It is seen that for the exponential $\operatorname{Exp}(-1.2 x)$ event, application criteria $K$ and $D$ decreases the maximum, shifting it to the region of lower values $D_{F}$. For the exponential
$\operatorname{Exp}(-0.35 x)$ events, application criterion $K$ leads to the formation of two new maxima instead of the initial one, and criterion $D$ suppresses distribution. Criterion $P$ suppresses the distribution for both types of exponential events. It is seen that, for the power law $\operatorname{Pow}(x,-2.04)$ events, the application of criteria $K$ and $D$ leads to a decrease in the maxima. For power $\operatorname{Pow}(x,-0.984)$ events, there is a decrease in maxima and suppression of the largest maximum when taking into account constraints $K$ and $D$, respectively. Criterion $P$ leads to the suppression of distributions for both types of power law events.

Thus, it was found that the criteria affect the shape of the distribution of background events in dimension $D_{F}$. This manifests itself in a decrease and shift in the distribution maxima or an almost complete suppression of background events. For fractals, in comparison with background events, the application of the introduced criteria does not entail a change in the shape of the distribution over $D_{F}$. 

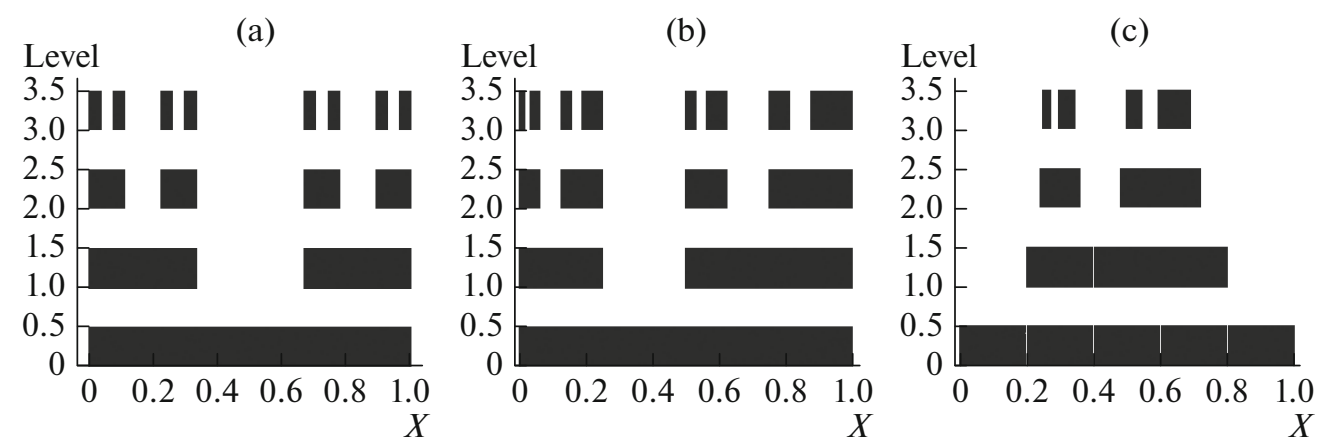

Fig. 9. Process of formation of fractals with independent (a), dependent (b), and combined (c) division.

\section{CONCLUSIONS}

In this paper, fractals and various background events (mixed, random, distributed according to Gauss's law, as well as according to power and exponential laws) are analyzed by $\mathrm{SePaC}$ method with aim to search for and reconstruct fractal structures.

It is shown that some of the background events are reconstructed as fractals. It was found that the suppression of power law and exponential events is less than that of mixed and random events, as well as events distributed over Gaussian.

Distributions of various events are considered for the dimensions of $D_{F}$. It was found that the distributions for fractals and background events are different. It is shown that the position of the maxima for mixed and random events depends on the multiplicity distribution, for exponential ones it depends on the value of the exponent parameter, and for events distributed over the Gaussian it does not depend on the value $\sigma$. The distribution of power law events has three maxima, the position of the first depends on the exponent and the positions of the others do not.

Values are proposed that take into account the features of fractal structures. A comparison of these values for fractals and background events is carried out. A significant difference in their behavior was established and criteria for background suppression were proposed.

The results of applying the criteria for various events are presented. It was found that the criteria affect the shape of the distribution of background events by dimension $D_{F}$. This manifests itself in a decrease and shift in the distribution maxima or an almost complete suppression of background events. Applying the criteria that are introduced for fractals does not entail a change in the distribution shape over $D_{F}$.

\section{APPENDIX A}

One distinctive feature of fractals is the difference between topological and fractal dimensions. The topological dimensions of a point, line, and plane are equal to zero, one, and two, respectively. The fractal dimension is determined by the dynamics of object formation. Fractals are characterized by different dimensions: Hausdorff-Besicovich, Minkowski (boxdimension), etc. [24, 25].

Hausdorff-Besicovitch dimension $D_{H}$ is called fractal and is defined as the value $d$ at which measure $M_{H}$ has a finite value

$$
M_{H}=\lim _{\epsilon \rightarrow 0} \inf _{C} \sum_{i=1}^{N} l_{i}^{d} .
$$

Here, operator inf means the minimum of all possible coverings $C$ of the considered set. $N$ is the number of probes with size $l_{i}<\epsilon$ covering the object. The quantity $\epsilon^{-1}$ is called the resolution.

Minkowski dimension (box dimension) $D_{b}$ is determined by the formula

$$
D_{b}=-\lim _{l \rightarrow 0} \frac{\ln N(l)}{\ln l} .
$$

Here $N(l)$ is the number of probes of size $l$ covering the object.

For fractals with independent division (Appendix B), the box dimension coincides with the HausdorffBesicovich dimension, and for fractals with dependent and combined division (Appendix B), they are different $\left(D_{b}>D_{H}\right)$. Box dimension is a rougher characteristic, since it assumes the same size of fractal elements at the same level.

\section{APPENDIX B}

In this paper, we consider various types of fractals (fractals with independent, dependent, and combined divisions), which are varieties of Cantor sets (Cantor dust). Fractals with dependent and combined division are called fractals with unequal ratios.

Consider fractals with independent division. The most famous representative of this type is the triadic Cantor set, the formation process of which is shown in Fig. 9a. The original element is a unit line [0;1] along 
the axis $X$. Let us conditionally divide it into three parts, corresponding to the formation of a fractal with a base $P_{F}=3$. Let us delete the second part and leave the first and third. Let us call this procedure the act of dividing a fractal element in the process of its formation. The resulting structure is denoted by $S=(101)$, where $\langle\langle 1\rangle\rangle$ denotes for the remainder and $\langle\langle 0\rangle\rangle$ denotes for the excluded. The number of units in the structure determines the number of new elements in each act of division. At the next levels, the division process is repeated. The new elements are the same size, equal to $1 / P_{F}$ the size of the parent, and are divided independently.

Figure $9 \mathrm{~b}$ shows the formation of a fractal with dependent division. Original element (line $[0 ; 1]$ ) is conventionally divided into four parts (base $P_{F}=4$ ). The first, third, and fourth parts remain at the first level (structure $S=(1011)$ ). At the next levels, the division process is repeated, taking into account that the segment consisting of the third and fourth parts represents a single object (dependent division). The number of new elements in each act of division is determined by the number of segments, not units in the structure. New elements differ in size $\left(1 / P_{F}\right.$ and $2 / P_{F}$ on the size of the parent).

The formation of a fractal with combined division is shown in Fig. 9c. The original element is conventionally divided into five parts $\left(P_{F}=5\right)$. The second, third, and fourth parts remain at the first level $(S=(0111))$, forming a segment. At the next levels, a combined division of the segment occurs; namely, the second part is divided independently, and the third and fourth as a single object (dependently). The resulting elements differ in size $\left(1 / P_{F}\right.$ and $2 / P_{F}$ on the size of the parent). The number of new elements in each division does not correspond to either the number of units in the structure or the number of segments. To determine them, a full enumeration of various options for selecting them from a segment is required. Note that the $\mathrm{SePaC}$ method tests hypotheses of independent and dependent division and approximately reconstructs fractals with combined division.

\section{REFERENCES}

1. T. Sjöstrand, S. Mrenna, and P. Skands, "PYTHIA 6.4 physics and manual," J. High Energy Phys. 0605, 026 (2006); hep-ph/0603175.

2. A. Bylinkin and A. Rostovtsev, "Parametrization of the shape of hadron-production spectra in high-energy particle interactions," Phys. At. Nucl. 75, 999 (2012).

3. A. Parvan, O. Teryaev, and J. Cleymans, "Systematic comparison of Tsallis statistics for charged pions produced in pp collisions," Eur. Phys. J. A 53, 102 (2017).

4. R. Ratha et al., "Event multiplicity, transverse momentum and energy dependence of charged particle production, and system thermodynamics in pp collisions at the large hadron collider," J. Phys. G 47, 0555111 (2020).
5. A. Khuntia et al., "Radial flow and differential freezeout in proton-proton collisions at $\sqrt{\mathrm{s}}=7 \mathrm{TeV}$ at the LHC," Eur. Phys. J. A 55, 3 (2019).

6. T. Bhattacharyya et al., "On the precise determination of the Tsallis parameters in proton-proton collisions at LHC," J. Phys. G 45, 055001 (2018).

7. C. Tsallis, "Possible generalization of BoltzmannGibbs statistics,” J. Stat. Phys. 52, 479-487 (1988).

8. C. Tsallis, "Nonextensive statistics: Theoretical, experimental and computational evidences and connections,” Braz. J. Phys. 29, 1-35 (1999).

9. M. Gell-Mann and C. Tsallis, Nonextensive Entropy: Interdisciplinary Applications (Oxford Univ. Press, USA, 2004).

10. A. Deppman, "Thermodynamics with fractal structure, Tsallis statistics, and hadrons," Phys. Rev. D 93, 054001 (2016).

11. A. Deppman, "Fractal structure of hadrons: Experimental and theoretical signatures," Universe 3, 62 (2017).

12. A. Deppman et al., "Fractal structure in Yang-Mills fields and non extensivity," arXiv: 1905.06382v1 [hepth] (2019).

13. A. Parvan, "Ultrarelativistic transverse momentum distribution of the Tsallis statistics," Eur. Phys. J. A 53, 53 (2017).

14. A. Parvan and T. Bhattacharyya, "Hadron transverse momentum distributions of the Tsallis normalized and unnormalized statistics,” Eur. Phys. J. A 56, 72 (2020).

15. N. Antoniou, A. Contogouris, C. Papadopoulos, and S. Vlassopulos, "Criticality, fractality and intermittency in strong interactions," Phys. Lett. B 245, 619 (1990).

16. H. Satz, "Intermittency and critical behavior," Nucl. Phys. B 326, 613-618 (1989).

17. T. Dedovich and M. Tokarev, "P-adic coverage method in fractal analysis of showers," Phys. Part. Nucl. Lett. 8, 521-532 (2011).

18. T. Dedovich and M. Tokarev, "Method of systems of the equations of p-adic coverages for fractal analysis of events," Phys. Part. Nucl. Lett. 9, 552-566 (2012).

19. T. Dedovich and M. Tokarev, "Comparision of fractal analysis methods in the study of fractals with independent branching," Phys. Part. Nucl. Lett. 10, 481-490 (2013).

20. T. Dedovich and M. Tokarev, "Analysis of fractal with dependent branching by box counting, p-adic coverages and system of equations of p-adic coverages," Phys. Part. Nucl. Lett. 10, 491-500 (2013).

21. T. Dedovich and M. Tokarev, "Analysis of fractals with combined partition,” Phys. Part. Nucl. Lett. 13, 169177 (2016).

22. T. Dedovich and M. Tokarev, "Reconstruction of the dimension of complete and incomplete fractals," Phys. Part. Nucl. Lett. 16, 240-250 (2019).

23. T. Dedovich and M. Tokarev, "A two-step procedure of fractal analysis," Phys. Part. Nucl. Lett. 13, 178-189 (2016).

24. B. Mandelbrot, The Fractal Geometry of Nature (Freeman, San Francisco, 1982).

25. J. Feder, Fractals (Plenum Press, New York, 1998).

26. T. Dedovich and M. Tokarev, "Fractal reconstruction in the presence of background," Phys. Part. Nucl. Lett. 14, 865-873 (2017). 